\title{
Deep superconducting magnetic traps for neutral atoms and molecules
}

\section{Citation}

Harris, J. G. E., R. A. Michniak, S. V. Nguyen, W. C. Campbell, D. Egorov, S. E. Maxwell, L. D. van Buuren, and J. M. Doyle. 2004. "Deep Superconducting Magnetic Traps for Neutral Atoms and Molecules." Review of Scientific Instruments 75 (1): 17. doi:10.1063/1.1633993.

\section{Published Version}

doi:10.1063/1.1633993

\section{Permanent link}

http://nrs.harvard.edu/urn-3:HUL.InstRepos:28424203

\section{Terms of Use}

This article was downloaded from Harvard University's DASH repository, and is made available under the terms and conditions applicable to Other Posted Material, as set forth at http:// nrs.harvard.edu/urn-3:HUL.InstRepos:dash.current.terms-of-use\#LAA

\section{Share Your Story}

The Harvard community has made this article openly available.

Please share how this access benefits you. Submit a story.

\section{Accessibility}




\title{
Deep superconducting magnetic traps for neutral atoms and molecules
}

\author{
J. G. E. Harris, ${ }^{\text {a) }}$ R. A. Michniak, and S. V. Nguyen \\ Harvard-MIT Center for Ultracold Atoms, Cambridge, Massachusetts 02138 and Department of Physics, \\ Harvard University, Cambridge, Massachusetts 02138 \\ W. C. Campbell, D. Egorov, S. E. Maxwell, and L. D. van Buuren \\ Department of Physics, Harvard University, Cambridge, Massachusetts 02138 \\ J. M. Doyle \\ Harvard-MIT Center for Ultracold Atoms, Cambridge, Massachusetts 02138 and Department of Physics, \\ Harvard University, Cambridge, Massachusetts 02138
}

(Received 22 August 2003; accepted 21 October 2003)

\begin{abstract}
We describe the design, construction and performance of three realizations of a high-field superconducting magnetic trap for neutral atoms and molecules. Each of these traps utilizes a pair of coaxial coils in the anti-Helmholtz geometry and achieves depths greater than $4 \mathrm{~T}$, allowing it to capture magnetic atoms and molecules cooled in a cryogenic buffer gas. Achieving this depth requires that the repulsive force between the coils (which can exceed 30 metric tons) be contained. We also describe additional features of the traps, including the elimination of trapped fluxes from the coils and the integration of the coils into a cryogenic vacuum environment suitable for producing cold atoms and molecules. (c) 2004 American Institute of Physics. [DOI: 10.1063/1.1633993]
\end{abstract}

\section{INTRODUCTION}

Magnetic traps can confine neutral atomic vapors away from material walls, thereby breaking thermal contact between the trapped atoms and their environment. This isolation makes it possible to cool the trapped atoms (e.g., by evaporative cooling) to temperatures well below what can be achieved otherwise, and has played a crucial role in the development of ultracold atomic physics and the attainment of Bose-Einstein condensation in dilute atomic gases.

In order for atoms to be confined in a magnetic trap, their thermal energy must be less than the potential energy barrier created by the magnetic fields of the trap. This means that the atoms' temperature $T$ and the depth of the trap $B_{\text {trap }}$ must satisfy $k_{B} T \ll \mu B_{\text {trap }}$ where $\mu$ is the atoms' magnetic moment and $k_{B}$ is Boltzmann's constant. However the magnetic traps that can be produced using current (or any foreseeable) technology are much shallower than the thermal energy typical of atoms in their equilibrium gas phase; as a result the atomic vapor must be cooled before it can be loaded into a magnetic trap.

Three types of cooling have been used to load atoms into magnetic traps. These approaches differ in the range of atomic species to which they are applicable, the maximum number of atoms they can load into a trap, and the lowest temperature to which they cool the atoms before loading. The most widely used method is laser cooling, in which atoms can be cooled to sub-mK temperatures. At these low temperatures, the atoms can be trapped by $\sim 10 \mathrm{mT}$ deep traps produced by copper coils in a room-temperature vacuum system. ${ }^{1}$ This approach is applicable only to atomic species with the appropriate optical properties, and is capable of loading $\sim 10^{10}$ atoms into a magnetic trap (limited by

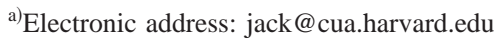

multiple scattering of photons from the cooling lasers, collisions between excited state atoms, and technical limits on the power of the cooling lasers). A second approach to loading cold atoms into magnetic traps (which is applicable only to atomic hydrogen) makes use of the unique properties of spinpolarized $\mathrm{H}$ in a He-coated chamber. The unusually low binding energy of $\mathrm{H}$ to $\mathrm{He}$ allows the $\mathrm{H}$ gas to be cooled to $\sim 100 \mathrm{mK}$ via contact with the He-coated walls. At this temperature the $\mathrm{H}$ can be trapped by $\sim 1 \mathrm{~T}$ magnetic fields produced by superconducting coils. ${ }^{2}$ As many as $10^{14} \mathrm{H}$ atoms can be magnetically trapped using this technique; however, the much higher binding energy of all other atom-surface pairs precludes the application of this approach to the trapping of any other atomic species.

A third approach, which is both applicable to a wide range of species and capable of loading large numbers of atoms, has been developed in our group. ${ }^{3}$ In this approach hot atoms or molecules (produced by a laser ablation pulse or an atomic/molecular beam ${ }^{4}$ ) are cooled by elastic collisions with a cryogenic $(T \sim 1 \mathrm{~K})$ helium buffer gas. After the atoms thermalize with the buffer gas they are cold enough to be trapped by magnetic fields of a few tesla, which are produced by superconducting coils. Unlike laser cooling or the cryogenic cooling of spin-polarized hydrogen, this technique should be applicable to any atomic or molecular species with a permanent magnetic moment and appropriate spinrelaxation cross-sections. It has been successfully used to load $\sim 10^{12}$ atoms of $\mathrm{Eu}, \mathrm{Cr}$, and $\mathrm{Mo}$, as well as $\sim 10^{8} \mathrm{~mol}-$ ecules of $\mathrm{CaH}$ into magnetic traps. ${ }^{5-8}$ However, the fact that the atoms are initially cooled to higher temperatures than in the cases of spin-polarized hydrogen or laser-cooled atoms has required the development of very deep magnetic traps ( $\sim 4 \mathrm{~T}$ deep) to ensure the condition $k_{B} T \ll \mu B_{\text {trap }}$ is met. 


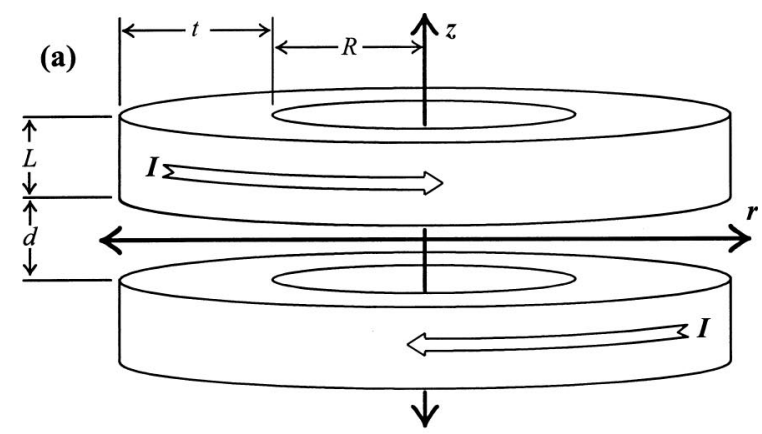

(b)

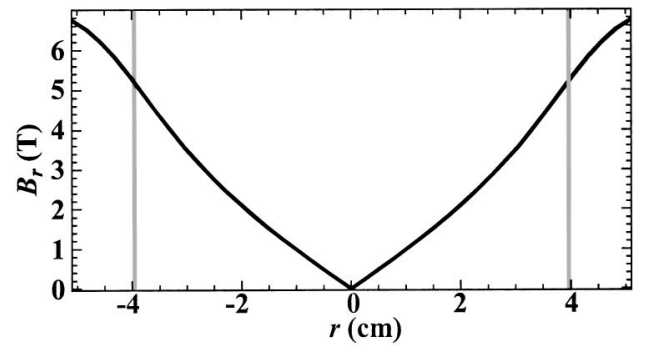

(c)

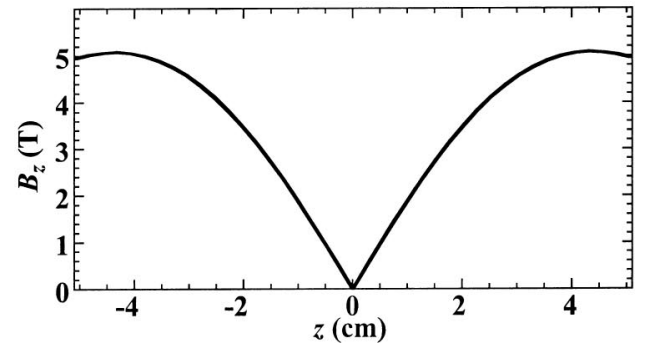

FIG. 1. (a) Schematic illustration of the anti-Helmholtz geometry. The coil dimensions $t, d, R$, and $L$ for each magnet are given in Table I. (b) Plot of the calculated $B_{r}(r, z=0)$ for the Mark 4 magnet at its maximum current. The gray lines indicate the walls of the magnet bore. (c) Plot of the calculated $B_{z}(r=0, z)$ for the Mark 4 magnet at its maximum current.

In this article, we describe the design, construction, and testing of these magnetic traps. We describe three different designs and focus on the depth of the traps produced by the superconducting coils, the coils' structural support, the optical access they provide, their integration into the surrounding cryogenic and vacuum environments, and the role of magnetic flux trapped within the coils. Last, we describe possible future directions which might improve the magnets' performance.

\section{MAGNET DESIGN}

In practice the deepest magnetic traps are produced by spherical quadrupole magnets, a geometry colloquially known as "anti-Helmholtz." These traps consist of a pair of identical coaxial coils whose currents $I$ are equal and flow in opposite directions, producing a magnetic field which is zero at the trap center and increases (to lowest order) as $|\vec{B}|$ $\propto \sqrt{4 z^{2}+r^{2}}$. Here $z$ is the coordinate along the axis of symmetry of the two coils and $r$ is the coordinate along the radial axis. Figure 1 shows a schematic of this geometry and the resulting fields. All the magnets described in this paper use the anti-Helmholtz geometry shown in Fig. 1. The difference between the smallest value of $|\vec{B}|$ (which is zero for antiHelmholtz traps) and the value of $|\vec{B}|$ where atoms can es- cape the trap (either via saddle points in $|\vec{B}|$ or by colliding with a material wall) sets the trap depth $B_{\text {trap }}$.

The maximum achievable $B_{\text {trap }}$ is limited by two factors: the critical current $I_{c}$ of the superconducting coils, and the repulsive force $F$ generated between the two coils. For the traps described here, $F$ can be greater than 30 metric tons. Thus the structural support of the coils is a crucial part of the overall magnet design. ${ }^{9}$ We first describe the general design considerations for the coils and their supports, and then provide a more detailed description of the individual magnet designs.

For each magnet design we calculate $B_{\text {trap }}$ and $F$ as follows: first we calculate the magnetic field profile $\vec{B}(z, r, I)$ for different values of the coil dimensions $t, d, R$, and $L$ (see Fig. 1) by numerically integrating the Biot-Savart equation. We then calculate $I_{c}$ for each coil by combining $\vec{B}(z, r, I)$ with the known magnetic field dependence of the shortsample critical current of the wire used in the coils. ${ }^{10}$ Last, we calculate $F$ for each pair of coils from

$$
F=2 \pi \frac{I_{c}}{A} \int_{R}^{R+t} d r \int_{d / 2}^{L+d / 2} d z \vec{B}\left(z, r, I_{c}\right) \cdot \vec{r}
$$

where $A=t L$ is the cross-sectional area of the coils.

The depth of the trap $B_{\text {trap }}$ depends upon $t, d, R$, and $L$ not only through their effect upon the magnetic field profile $|\vec{B}(z, r, I)|$, but also through their effect upon $I_{c}$ and $F$. The combination of these effects leads to a complicated dependence of $B_{\text {trap }}$ on $t, d, R$, and $L$. We find that for the range of geometries considered, $B_{\text {trap }}$ generally increases with the cross-sectional area $A$ of the coils and decreases with the separation $d$. However $F$ also increases rapidly with $A$, limiting the maximum practical size of the coils.

The maximum $F$ which can be tolerated (and hence the optimal coil geometry and trap depth) is determined by the tensile strength of the structure which holds the two coils together. The support structure material must possess high tensile strength and toughness against brittle failure and fatigue at cryogenic temperatures. In order to preserve the spatial profile of the trapping fields, it also needs to be nonsuperconducting and non-magnetic at $4.2 \mathrm{~K}$ after repeated cycling of temperature, magnetic field, and mechanical stress. The support structures for all three magnets discussed in this article are machined from Grade 6 titanium alloy (5\% $\mathrm{Sn}, 2.5 \% \mathrm{Al}$ ), which meets these requirements. ${ }^{11}$ Grade 5 titanium alloy $(6 \% \mathrm{Al}, 4 \% \mathrm{~V})$ is more readily available and possesses comparable mechanical properties, but is superconducting at $4.2 \mathrm{~K}^{12}$

The support structure is designed to be conceptually simple in order to avoid unforeseen stress concentrations which could lead to catastrophic mechanical failure. Where practical, we have used finite element analysis to calculate stress concentrations. The dimensions of the support structure are chosen so that the tensile stress produced by $F$ (ignoring stress concentrations) is at all points in the structure a factor of six below the room temperature tensile yield stress. Assuming that the stress concentration factor is nowhere greater than three, this leaves a factor-of-two margin of safety. An additional margin of safety is provided by the fact 
(a)

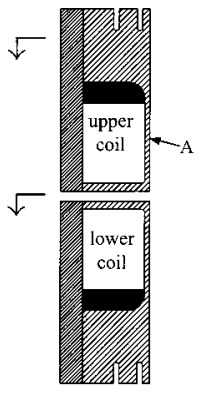

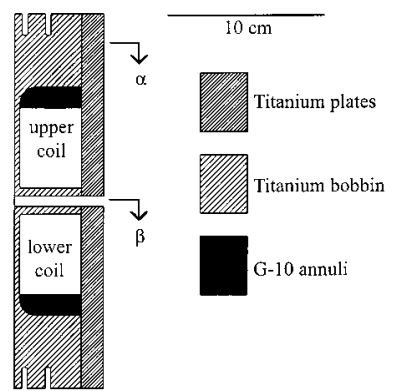
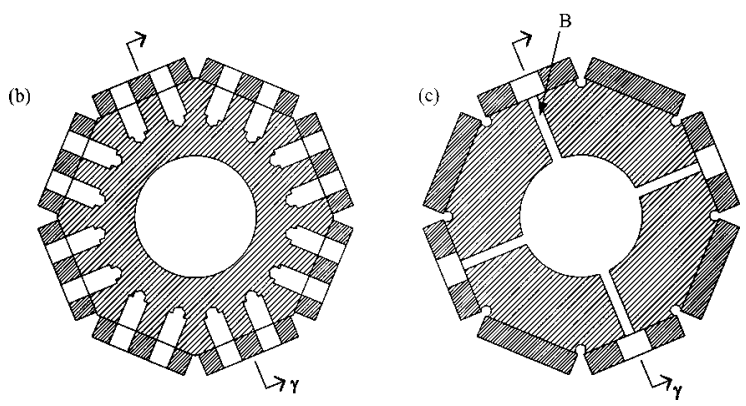

FIG. 2. (a) Cross-sectional view of the Mark 3 magnet. The magnet coils are wound on the titanium bobbin. The eight titanium plates provide additional support against the intercoil force at the outer diameter of the magnet. Each plate is attached to the bobbin by four $12.7 \mathrm{~mm}$ diameter titanium pegs. The thinnest portion of the bobbin wall is indicated by $A$. (b) Cross section along $\alpha$, showing the 16 peg holes used to attach the 8 plates to the upper portion of the bobbin. The recess at the bottom of each peg hole allows gases trapped behind each peg to be vented out the top of the bobbin. For clarity, the pegs are omitted. $\gamma$ indicates the cross section shown in (a). (c) Cross section along $\beta$, the midplane of the magnet, showing the part of the bobbin which separates the two coils. Also shown are the four optical access holes (one of which is labeled $B$ ) which penetrate from the outer to the inner diameter of the bobbin. The rounded notches at the corners of the midplane provide room for the magnet leads.

the tensile yield strength of Grade 6 titanium increases by a factor of $\sim 1.6$ between room temperature and $4.2 \mathrm{~K}$ while maintaining reasonable ductility. ${ }^{11}$

\section{A. Mark 3 magnet}

Figure 2 shows a cross-sectional view of an antiHelmholtz magnet used to trap atomic $\mathrm{Cr}$ and Mo. This magnet (referred to as the Mark 3) is cooled to $4.2 \mathrm{~K}$ in a liquid helium bath. Both the outer and inner surfaces of the magnet are in contact with the liquid helium. The trapped atoms are stored in a vacuum can which fits inside the inner diameter of the magnet bore. The dimensions of the coils are given in Table I. The coils are wound with 8266 turns each of Formvar-insulated $\mathrm{NbTi} / \mathrm{Cu}$ wires (Supercon 54S wire: 0.016 " outer diameter, 54 filaments of $\mathrm{Nb}_{0.52} \mathrm{Ti}_{0.48}$ embedded in a copper matrix, 1:1.3 superconducting-to-normal ratio). The critical current of the coils as a whole is calculated to be
$I_{c}=76 \mathrm{~A}$. At this current, the coils produce a trapping field which increases from zero at the center of the magnet to $B_{\text {trap }}=4.43 \mathrm{~T}$ at the inner surface of the magnet bore $(z$ $=0 \mathrm{~mm}, r=38.1 \mathrm{~mm})$. Along the axis of symmetry of the magnet, the magnetic field reaches a saddle point at $z$ $= \pm 43.9 \mathrm{~mm}, r=0 \mathrm{~m}$, at which $\vec{B}= \pm 4.77 \hat{z} \mathrm{~T}$. In all the traps described here, the magnetic field at these saddle points is larger than the field at the inner surface of the magnet; hence, it is the latter which sets $B_{\text {trap }}$.

When this magnet is fully energized (i.e., $I=I_{c}$ ), $F$ $=2.3 \times 10^{5} \mathrm{~N}$ (23.5 metric tons). The support against $F$ for this magnet is provided in part by the titanium bobbin upon which the coils are wound. At their narrowest (point $A$ in Fig. 2 ), the walls of this bobbin are $3.18 \mathrm{~mm}$ thick, with a crosssectional area of $780 \mathrm{~mm}^{2}$. Transitions in the dimensions of the bobbin are made gradually to avoid stress concentrations. For example, the thick uppermost and lowermost portions of the bobbin merge into the thin-walled portions with a radius of curvature of $10 \mathrm{~mm}$. Finite element analysis indicates that this transition produces a stress concentration factor of $\sim 1.5$.

Additional support is provided by eight plates external to the coils. Each of these 12.7-mm-thick plates is secured to the upper and lower portions of the bobbin by four 12.7mm-diameter pegs. The 8 plates and 32 pegs are machined from the same titanium alloy as the bobbin. While the inner bobbin is nominally sufficient to restrain the coils against $F$, these plates provide support at the outer diameter of the coils and ensure that the margin of safety described above is maintained.

Optical access to the trapping region along the axial direction is provided by the $76.5 \mathrm{~mm}$ bore of the magnet. Four 6.3-mm-diameter holes dilled in the midplane of the magnet (points $B$ in Fig. 2) provide additional radial access.

\section{B. Mark 4 magnet}

Figure 3 shows a cross-sectional view of a different antiHelmholtz trap (the Mark 4). Its dimensions and other specifications are listed in Table I. In this design the coils are wound on a thin-walled (1.65 mm thick) brass bobbin which is not intended to provide any support against $F$. Instead, support is provided by a cylindrical titanium cask external to the coils. The coils are mechanically linked to the cask by a pair of titanium annuli above the upper coil and below the lower coil (points $A$ in Fig. 3) by 12 pegs each of $20.64 \mathrm{~mm}$ diameter. The shear strength of these pegs (assumed to be $60 \%$ of their tensile strength) and the calculated $I_{c}$ set nearly identical limits to $B_{\text {trap }}$ for this magnet.

TABLE I. The dimensions of the coils (as defined in Fig. 1), the number of turns in each coil, the superconducting-to-normal metal ratio of the wire used, the critical current $I_{c}$, the repulsive intercoil force $F$, and the depth $B_{\text {trap }}$ for each of the magnetic traps discussed in the text.

\begin{tabular}{|c|c|c|c|c|c|c|c|c|c|}
\hline & $L(\mathrm{~mm})$ & $d(\mathrm{~mm})$ & $t(\mathrm{~mm})$ & $R(\mathrm{~mm})$ & $\begin{array}{l}\text { Number } \\
\text { of turns }\end{array}$ & $\begin{array}{l}\text { s:n ratio } \\
\text { of wire }\end{array}$ & $I_{c}(\mathrm{~A})$ & $F(\mathrm{~N})$ & $B_{\text {trap }}(\mathrm{T})$ \\
\hline Mark 3 & 50.67 & 20.14 & 31.63 & 41.40 & 8266 & $1: 1.3$ & 78.5 & $2.3 \times 10^{5}$ & 4.43 \\
\hline Mark 4 & 61.0 & 15.24 & 35.56 & 42.16 & 7520 & $1: 1.3$ & 99 & $3.1 \times 10^{5}$ & 4.72 \\
\hline Mark 5 & 63.5 & 15.24 & 33.59 & 41.21 & 4745 & $1: 2$ & 145 & $2.7 \times 10^{5}$ & 4.36 \\
\hline
\end{tabular}


(a)

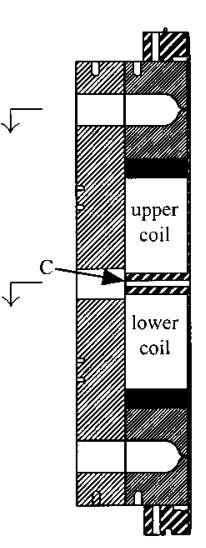

(b)

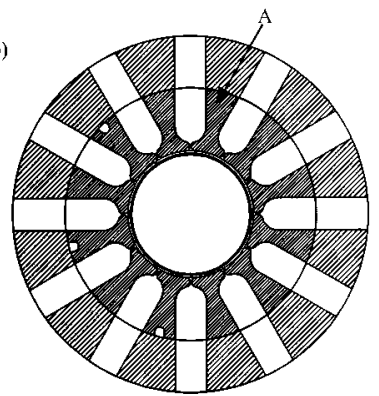

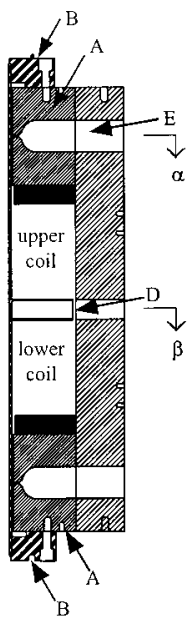

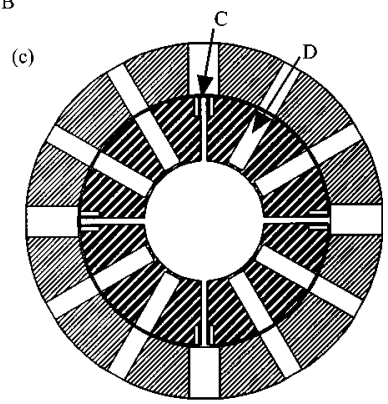

FIG. 3. (a) Cross-sectional view of the Mark 4 magnet. The titanium annuli $(A)$ and the G-10 annuli are slid over the brass bobbin and then captured by brazing the brass vacuum flanges $(B)$ to the bobbin. (b) Cross section along $\alpha$, showing the twelve 20.64-mm-diameter holes in the titanium annuli and cask into which the load-bearing pegs are inserted. For clarity, the pegs are omitted. The peg holes and the other blind holes have round bottoms in order to avoid stress concentrations. Each peg hole also has a $1 \mathrm{~mm}$ diameter hole which extends to the inner diameter of the annulus in order to vent any gases trapped by the pegs. The coil leads enter the magnet via the three notches on the outer diameter of the bobbin. (c) Cross section along $\beta$, the midplane of the magnet, showing the part of the brass bobbin which separates the two coils. One of the four optical ports is indicated by $C$. At each optical port the outer diameter of the brass bobbin is faced off to provide a flat surface for an indium seal, allowing the magnet bobbin to serve as a vacuum can. One of the eight radial ports is indicated by $D$. These ports do not penetrate into the bore of the magnet.

The support structure for this magnet can be substantially thicker than in the Mark 3 design because the cask is external to the coils. This provides a twofold advantage with regard to $B_{\text {trap }}$ : a greater $F$ can be tolerated, and the inner wall of the magnet can be thinner. The latter allows the trapping volume of the atoms to extend closer to the coils themselves, making more efficient use of the magnetic fields (see Fig. 1). Still more efficient use of the fields can be made by removing the necessity of an additional vacuum can inside the magnet bore. This is achieved in the Mark 4 by having the magnet bobbin serve as a vacuum can. Brass indium-seal flanges (points $B$ in Fig. 3) are brazed onto the brass bobbin and can connect with the vacuum spaces of the rest of the experiment. In this configuration, only the outer surface of the magnet is in direct contact with the liquid helium bath.

The midplane of the "Mark 4" contains four radial optical access ports (points $C$ in Fig. 3) which allow for windows to be mounted with indium seals (in order to preserve the vacuum in the magnet bore). Eight additional radial ports (points $D$ in Fig. 3) extend close to the inner wall of the

(a)

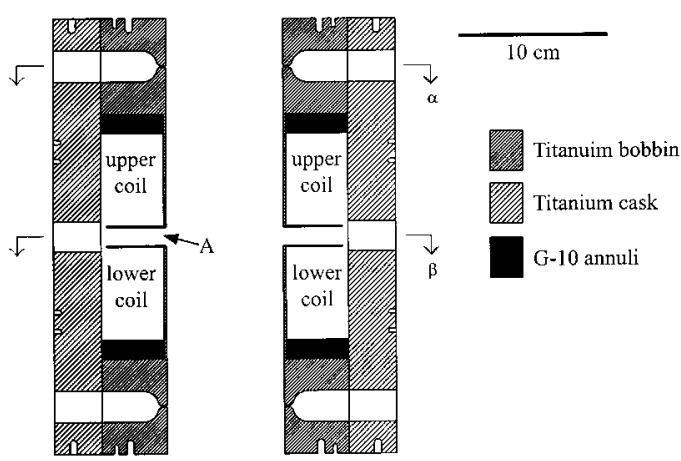

(b)
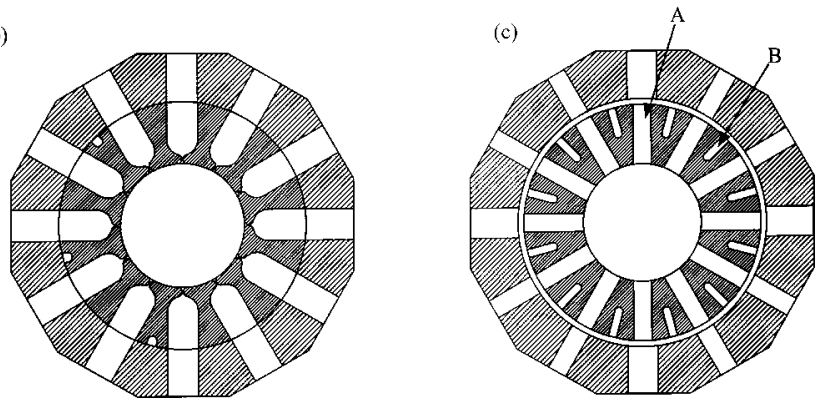

FIG. 4. (a) Cross-sectional view of the Mark 5 magnet. The magnet coils are wound on the titanium bobbin. Support against the intercoil force is provided by the titanium cask which is external to the coils and linked to the bobbin by 24 titanium pegs. (b) Cross section along $\alpha$, showing the 12 20.64-mm-diameter holes in the titanium annuli and cask into which the load-bearing pegs are inserted. For clarity, the pegs are omitted. The peg holes and the other blind holes have round bottoms in order to avoid stress concentrations. Each peg hole also has a 1-mm-diameter hole which extends to the inner diameter of the bobbin in order to vent any gases trapped by the pegs. The coil leads enter the magnet via the three notches on the outer diameter of the annuli. (c) Cross section along $\beta$, the midplane of the magnet, showing the part of the titanium bobbin which separates the magnet coils. Also shown are the 12 radial access ports (one is indicated by A) which penetrate from the outer to the inner diameter of the bobbin. The 12 blind holes (one is indicated by B) allow copper cooling strips to be anchored to the magnet midplane.

bobbin (but not into the vacuum space). These ports may be used to bring additional coils close to the trapping region in order to perturb the trapping fields in a controlled fashion. Similar geometries have been used in other labs to remove the region of zero magnetic field from the trap. ${ }^{13}$ This has been crucial to avoid Majorana spin-flip losses of trapped atoms at very low temperatures. ${ }^{14}$

An additional refinement in this design is the inclusion of resistive wire wound on the inner and outer diameters of both superconducting coils. This wire serves as a heater, and can be used to drive the superconducting coils normal, thereby removing any trapped fluxes from the coils. The advantage of this option is described in Sec. IV.

\section{Mark 5 magnet}

Figure 4 shows a cross-sectional view of a third magnet design (the Mark 5), which incorporates a number of features from both of the previous designs. Here the coils are wound on a thin-walled titanium bobbin which is not intended to provide support against $F$. As in the Mark $4, F$ is transferred to an external cask via 12 pegs each in the upper and lowermost parts of the bobbin. Unlike either the Mark 3 or the 
Mark 4, the Mark 5 is designed to operate in vacuum as part of a molecular beam apparatus (again removing the need for an additional vacuum can interior to the magnet bore). In the absence of direct contact with liquid helium, cooling of the coils is ensured by copper strips which improve the thermal conductance between the coils and the bobbin. The bobbin is in turn cooled via several 25.4-mm-diameter copper braids attached to the $4.2 \mathrm{~K}$ cold plate of a liquid helium Dewar. ${ }^{15}$ This design provides radial optical access through all 12 ports in the bobbin midplane (points $A$ in Fig. 4).

\section{MAGNET CONSTRUCTION}

Because the mechanical support of the energized coils is crucial to their performance, a sample from each piece of Grade 6 titanium alloy stock used in the construction is sent to an independent facility for testing of both chemical content and room temperature mechanical properties. If the stock material meets the specifications of ASTM B348 Grade 6, the various parts are machined at the Division of Engineering and Applied Sciences Machine Shop, Harvard University, Cambridge, MA. The bobbin is then sent to American Magnetics, Inc., Oak Ridge, TN where the coils are wound. Final assembly (i.e., of the external support cask) is performed at Harvard.

In the case of the Mark 3 magnet, the titanium bobbin and plates were first machined without the peg holes shown in Fig. 2(b). Then the plates were attached to the bobbin and the peg holes drilled (simultaneously in both the bobbin and the plates) to slightly under their final $12.7 \mathrm{~mm}$ diameter. The side plates were then removed so that the G-10 annuli could be put in place and the coils wound. After the winding, the pegs were machined and the side plates were reattached to the bobbin. Finally, the peg holes were reamed out to accept the pegs with $60 \mu \mathrm{m}$ radial clearance. Machining the final diameter of the peg holes in both the bobbin and the plates simultaneously while they are bolted together helps ensure that the pegs achieve the best possible fit.

Because this stage of the machining is done on the assembly containing the coils, care must be taken to ensure that the coils are not damaged. In order to avoid trapping machine oil in the coils, either isopropanol or ethanol is used as a coolant for the machining (on the grounds that they will not attack the epoxy in which the coils are impregnated, and should eventually evaporate).

For the Mark 4 design, the coils were wound on the brass bobbin with the titanium annuli and G-10 annuli secured in place (these annuli are captured by the indium-seal flanges mentioned above). The external titanium cask was then slid over this assembly (the annuli and cask were machined for a nominal $50 \mu \mathrm{m}$ radial gap). Four temporary pegs were inserted in the peg holes in the titanium cask and annuli (initially drilled to be $19.05 \mathrm{~mm}$ in diameter) to lock the cask in place relative to the annuli and coils. Then the peg holes were machined out to their full diameter of 20.64 $\mathrm{mm}$. Each titanium peg was machined to fit into its hole with a nominal clearance of $5 \mu \mathrm{m}$. With this clearance the pegs slide smoothly into their holes. A similar procedure was used with the Mark 5 design.

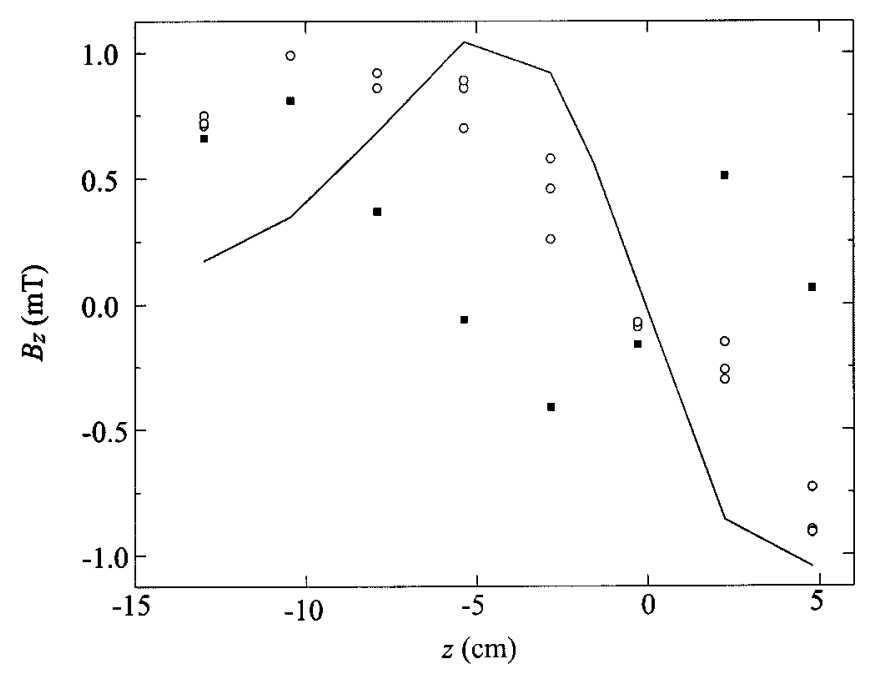

FIG. 5. Plots of $B_{z}$, the $z$-component of the magnetic field measured along the $z$-axis of the Mark 3 magnet. The open points show the remnant $B_{z}$ after the magnet has been ramped from 50 to $0 \mathrm{~A}$ (data from three separate rampdowns are shown). The solid points show $B_{z}$ measured after the current has been ramped from 50 to $0 \mathrm{~A}$ and a small negative current is run through the coils in an attempt to cancel the residual field. For comparison, the solid line shows $B_{z} / 3000$ with $50 \mathrm{~A}$ in the magnet.

\section{MAGNET PERFORMANCE}

The Mark 3 magnet reached its calculated $I_{c}$ (76 amps) with very little training. It has been used to trap atomic $\mathrm{Cr}$ and Mo. The Zeeman-broadening of the absorption line shapes of these trapped atoms is consistent with the magnet's calculated field profile. ${ }^{6}$

The Mark 3 has also been used to evaporatively cool atomic $\mathrm{Cr}$ and Mo. This is achieved by initially trapping the atoms in a deep magnetic trap (i.e., $I \approx I_{c}$ ) and then ramping $I$ down. As $I$ decreases and the magnetic trap becomes shallower, the atoms cool via evaporation over the edge of the trap and also via adiabatic expansion. However it is observed that when $I$ is ramped from near its maximum value to less than $0.3 \mathrm{~A}$ the magnetic field in the trapping region no longer scales with $I$, and does not vanish when $I=0$ A. Figure 5 shows a plot of the $\hat{z}$ component of $\vec{B}$ measured along the $z$-axis using a cryogenic Hall probe after $I$ has been ramped from 50 to $0 \mathrm{~A}$. This remnant field is attributed to trapped fluxes in the superconducting wire, and is typically $\sim 1 \mathrm{mT}$. The spatial dependence of this remnant magnetic field is not identical to that produced by current in the coils, and so cannot be canceled by reversing the current in the coils, as shown in Fig. 5. This remnant field strongly perturbs the shape of the trap at low I, complicating the spectroscopy of the trapped atoms and limiting the progress of their evaporative cooling. ${ }^{6,7}$ For this reason, it is desirable to remove these trapped fluxes.

The Mark 4 magnet reached its calculated $I_{c}$ (99 amps) without any quenching when tested with both the inner and outer surfaces of the magnet immersed in liquid helium (i.e., with the bore not under vacuum). With the bore under vacuum, the magnet reached 70 amps after a small amount of training. However, energizing the coils above about 40 amps caused the brass bobbin to leak, precluding its use as a vacuum space. This leak (which only appeared when charg- 


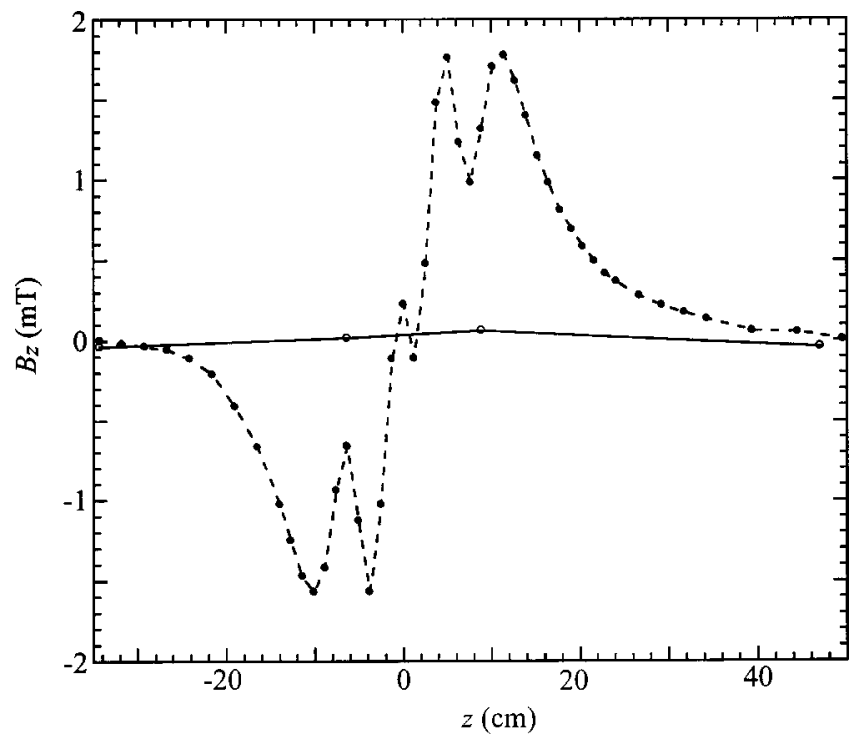

FIG. 6. The solid points show the remnant field $B$ zeasured along the $z$-axis of the Mark 4 magnet after ramping from 98 to $0 \mathrm{~A}$ (note the different horizontal scale from Fig. 3). The open points show $B_{z}$ measured after the integrated heater wires are used to drive the magnet coils normal and then the coils are allowed to cool back to $4.2 \mathrm{~K}$. A linear background of $9 \mu \mathrm{T} / \mathrm{cm}$ has been subtracted from both data sets. The lines are guides to the eye.

ing the magnet) may be due in part to the fact that the differential thermal contraction of the brass bobbin and the titanium sheath is such that cooling the assembly from 300 to $4.2 \mathrm{~K}$ produces a stress comparable to the room temperature tensile yield stress of brass. Presumably this problem could be solved in future designs by machining the bobbin from the same titanium alloy as the outer sheath. By using a vacuum can inside the magnet bobbin similar to the Mark 3 arrangement, we have also used this magnet to trap and evaporatively cool atomic $\mathrm{Cr}$, and achieved similar results to those described in Ref. 6. With this arrangement the magnet reached its full $I_{c}$.

The ability of the heater coils wound into the Mark 4 magnet to remove trapped fluxes is demonstrated in Fig. 6. Figure 6 (solid points) shows $\vec{B}_{z}$ measured along the $z$ axis after $I$ has been ramped from 98 down to 0 A. Figure 6 (open points) shows $\vec{B}_{z}$ measured after the coils have been heated above their superconducting transition temperature and allowed to return to $4.2 \mathrm{~K}$. This test was performed with both the inner and outer surfaces of the magnet immersed in liquid helium.

The Mark 5 magnet reached its calculated $I_{c}$ (145 amps) both while immersed in liquid helium and while operated in vacuum. In each case the magnet quenched three times at $\sim 60 \mathrm{~A}$ before reaching its full $I_{c}$. For the in-vacuum tests, the magnet and its leads were carefully heat sunk to the cold plate of a helium Dewar. The magnet leads were fed through the liquid helium bath of the Dewar and thence into the vacuum space of the Dewar to the magnet.

\section{FUTURE DIRECTIONS}

The efficiency with which atoms and molecules cooled by a cryogenic buffer gas can be magnetically trapped depends to a large degree upon the depth of the magnetic trap

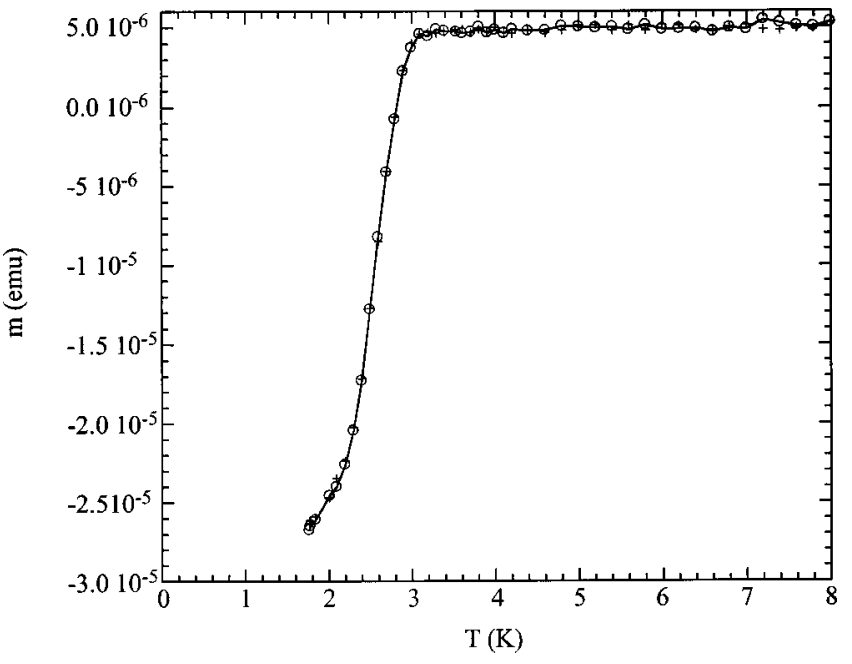

FIG. 7. Magnetic moment $m$ as a function of temperature of a $15.1 \mathrm{~mm}^{3}$ sample of Grade 6 titanium alloy in an applied field of $1 \mathrm{mT}$. The superconducting transition (evidenced by the onset of diamagnetism) can be seen at $\sim 3$ K. Open symbols: zero field cooled data. Crosses: field cooled data.

they are loaded into. As a starting point for considering possible future increases in the depth of magnetic traps, we note that for the Mark 4 design, the critical current of the superconducting coils and the maximum allowable repulsive force between the coils is reached at roughly the same current. Thus further improvement will require improving both the strength of the support structure and the performance of the superconducting coils.

At present the maximum $F$ is limited by the shear strength of the pegs. However substantial scaling up of the peg size would necessitate their merging, as can be seen, e.g., from Fig. 4(b). While this may be practical, it would represent an increased complexity in the design, stress analysis, and manufacture of the magnets. A more promising route would be the use of higher strength materials. Nominally nonmagnetic steel alloys with more than twice the lowtemperature tensile yield strength of Grade 6 titanium (such as MP35 $\mathrm{N}^{16}$ ) have been proposed for use in the construction of high-field solenoids, and may be applicable to neutral particle traps as well. While their excellent mechanical properties could allow larger values of $F$, the magnetic and superconducting properties of the material at $4.2 \mathrm{~K}$ after repeated cycles of magnetic field, temperature and stress are not known.

A possible route to an increased $I_{c}$ (and hence $B_{\text {trap }}$ ) would be to replace the $\mathrm{NbTi}$ wire (used in all the coils described here) with $\mathrm{Nb}_{3} \mathrm{Sn}$, either in part or in whole. The use of $\mathrm{Nb}_{3} \mathrm{Sn}$ coils in solenoids approximately doubles the magnetic fields which can be achieved. Whether or not such gains could be achieved in the anti-Helmholtz configuration will depend upon the relative brittleness of $\mathrm{Nb}_{3} \mathrm{Sn}$ compared to $\mathrm{NbTi}$, and the increased complexity of producing the coils.

More modest gains in $I_{c}$ could be realized with $\mathrm{NbTi}$ magnets by cooling the coils below $4.2 \mathrm{~K}$. However, our measurements of Grade 6 titanium alloy indicate that it superconducts below $T_{c}=3.0 \mathrm{~K}$. This is shown in Fig. 7 which plots the measured magnetic moment of a sample of Grade 6 alloy as a function of temperature in an applied field of $1 \mathrm{mT}$. 
As result of this superconducting transition, magnet temperatures much below $4.2 \mathrm{~K}$ would also necessitate the use of a different (i.e., nonsuperconducting) material for the support structure in applications which required the magnet to be run at low currents (i.e., evaporative cooling by lowering the trap depth). At higher currents the magnetic field produced by the coils will drive the support structure normal. ${ }^{12}$

The depth of the magnetic trap is not the only parameter which limits its effectiveness, however. For evaporative cooling to the lowest temperatures, it is also necessary to produce a trap which does not have a point of $|\vec{B}|=0$. While the radial ports described above offer the possibility of achieving this, other approaches might profitably be integrated into the structure of the magnet.

\section{ACKNOWLEDGMENTS}

This work was supported through the NSF Center for Ultracold Atoms. We gratefully acknowledge the machining expertise of Louis DeFeo and Richard Anderson of the Harvard University Division of Engineering and Applied Sciences Machine Shop.

${ }^{1}$ C. E. Wieman, D. E. Pritchard, and D. J. Wineland, Rev. Mod. Phys. 71, S253 (1999).

${ }^{2}$ H. F. Hess, G. P. Kochanski, J. M. Doyle, N. Masuhara, D. Kleppner, and T. J. Greytak, Phys. Rev. Lett. 59, 672 (1987).

${ }^{3}$ J. M. Doyle, B. Friedrich, J. Kim, and D. Patterson, Phys. Rev. A 52, R2515 (1995).

${ }^{4}$ D. Egorov, T. Lahaye, W. Schollkopf, B. Friedrich, and J. M. Doyle, Phys. Rev. A 67, 043401 (2003).

${ }^{5}$ J. D. Weinstein, R. deCarvalho, J. Kim, D. Patterson, B. Friedrich, and J. M. Doyle, Phys. Rev. A 57, R3173 (1998).
${ }^{6}$ J. D. Weinstein, R. deCarvalho, C. I. Hancox, and J. M. Doyle, Phys. Rev. A 65, 021604 (2002).

${ }^{7}$ R. deCarvalho, C. I. Hancox, and J. M. Doyle, J. Opt. Soc. Am. 20, 1131 (2003).

${ }^{8}$ J. D. Weinstein, R. deCarvalho, T. Guillet, B. Friedrich, and J. M. Doyle, Nature (London) 395, 148 (1998).

${ }^{9}$ The force we are concerned with here is the intercoil force (which by symmetry is directed along the $z$ axis of Fig. 1(a). The intracoil forces are comparable to those in standard solenoids and are contained by the tensile strength of the wire and the epoxy in which the wire is potted.

${ }^{10}$ Martin N. Wilson, Superconducting Magnets (Oxford Science Publications, Oxford, 1997).

${ }^{11}$ K. Nagai, K. Ishikawa, T. Mizoguchi, and Y. Ito, Cryogenics 26, 19 (1986); K. Ishikawa, K. Nagai, K. Nagai, T. Yuri, O. Umezawa, and K. Ishikawa, Proceedings 11th International Conference on Magnet. Tech. Vol. 1, edited by T. Sekiguchi and S. Shimimato (Elsevier, New York, 1990), pp. 754-759; O. Umezawa, K. Nagai, and K. Ishikawa, Mater. Sci. Eng., A 129, 217 (1990); K. Nagai, O. Umezawa, T. Yuri, and K. Ishikawa, Eng. Fract. Mech. 40, 957 (1991); O. Umezawa, T. Yuri, and T. Ogata, JSME Int. J., Ser. A 34, 347 (1991); O. Umezawa and K. Ishikawa, Cryogenics 32, 873 (1992).

${ }^{12}$ The superconductivity observed in magnet structures fabricated from Grade 5 alloy titanium (not described in this article) is suppressed by the magnetic fields generated when the magnet is fully energized. However as the magnet current is decreased (necessary for evaporative cooling of the trapped atoms), the superconductivity reappears, expelling magnetic flux from the trapping region in an unpredictable fashion and degrading the quality of the trap.

${ }^{13}$ T. Esslinger, I. Bloch, and T. W. Hänsch, Phys. Rev. A 58, R2664 (1998).

${ }^{14}$ W. Petrich, M. H. Anderson, J. R. Ensher, and E. A. Cornell, Phys. Rev. Lett. 74, 3352 (1995).

${ }^{15}$ American Magnetics Inc., Oak Ridge, TN, cryogen-free magnet technology.

${ }^{16}$ B. Fultz, A. DuBois, H. J. Kim, and J. W. Morris, Jr. Cryogenics 24, 687 (1984). 